\title{
Estudo da dirofilariose em gatos no Engenho do Mato, região oceânica de Niterói-RJ, Brasil
}

\section{Study of dirofilariasis in cats of the Engenho do Mato, oceanic region of Niterói-RJ, Brasil}

\author{
Luciana Almeida Martins Gomes, ${ }^{\star}$ Maria Lucia Serrão, ${ }^{\star \star}$ Claudia Dantas, ${ }^{\star \star \star}$ Norma Labarthe ${ }^{\star \star \star \star}$
}

\begin{abstract}
Resumo
A dirofilariose é uma doença que se apresenta distribuída mundialmente, sendo causada pelo nematóide Dirofilaria immitis (Leidy, 1856) que acomete várias espécies animais. Nos gatos a microfilaremia é inexistente ou de duração muito curta, há grande quantidade de migrações erráticas e curas espontâneas. Os sintomas, quando presentes, freqüentemente são mais utilizados testes laboratoriais, diagnóstico da dirofilariose felina é mais difícil de ser estabelecido. No entanto, podem ser conhecer-se a prevalência dessa área. Foi realizado o teste de Knott anticorpos nos gatos. Dentre os cães observou-se presença de anticorpos nos gatos.
\end{abstract}

Palavras-chave: Dirofilaria immitis, dirofilariose, diagnóstico

\begin{abstract}
Heartworm disease is worldwide distributed and it's etiologic agent is the nematode Dirofilaria immitis (Leidy, 1856) which infect many animal species. In cats microfilaremia is inexistent or short lived, there are many erratic migrations and self cures and clinical signs, when present, are usually more severe than in dogs. Diagnosis of feline heartworm is difficult to be established but it can be done by antigen or antibody tests. To know the prevalence of canine and feline heartworm in Engenho do Mato, microfilaria diagnosis by Knott's modified and dogs were collected in this area. The canine blood samples were collected for $26 \%(20 / 77)$ were microfilaremic and none of the cats showed antibodies.
\end{abstract}

Keywords: Dirofilaria immitis, heartworm, diagnosis

\section{Introdução}

Dirofilariose é uma doença que tem o nematóide Dirofilaria immitis (Leidy, 1856; Railliet e Henry, 1911) como agente etiológico. Apresenta ampla distribuição geográfica e, embora a ocorrência felina seja menor que a canina, há relatos de infecções felinas por $D$. immitis em várias partes do mundo, sendo a maioria deles achados isolados (Guerrero et al., 1992; McCall et al., 1994). O número de casos de dirofilariose felina vem aumentando e este parasitismo assinalado em novas regiões. Esse aumento pode estar relacionado com: 1) a maior utilização dos métodos diagnósticos (McCall et al., 1994; Brown et al., 1999) devido à redução do custo, praticidade e melhor eficiência dos testes (Knight et al., 1999); 2) a mobilidade dos animais, viajando freqüentemente; 3) mudança de hábitos alimentares dos mosquitos ou 4) pela associação de vários desses fatores (Brown et al., 1999).
Além de ser pouco freqüente, o diagnóstico da dirofilariose felina é mais difícil que o da canina porque a pesquisa de microfilárias é ineficiente, embora a microfilaremia, quando presente, constitua-se em diagnóstico definitivo (Holmes, 1993; McCall et al., 1994; Atkins et al., 1995; McCall et al. 1995; Mansour et al., 1995; Ryan et al., 1995; Dillon et al., 1997b; Atkins et al., 1997; Schrey e Trautvetter, 1998). Felinos apresentam cargas parasitárias muito baixas quando comparadas às caninas, e a concentração de antígenos encontra-se abaixo da sensibilidade da maioria dos testes (Tonelli, 1989; Holmes, 1993; Dillon et al., 1996; Atkins et al., 1997) simulando uma freqüência ainda mais baixa do que a provável realidade.

Alguns sintomas podem aparecer 3 a 4 meses após a infecção, quando a maioria dos testes apresenta resultado negativo (Dillon et al., 1996). Alterações radiográficas podem ser

\footnotetext{
- Mestranda CPG Clínica Médica Veterinária UFF. Rua Itaocara 88 Santa Rosa, Niterói, RJ. CEP 24240-100. E-mail: cmgomes @cruiser.com.br.

** Mestranda CPG Biologia Parasitária 1OC, Fiocruz. Rua Domingues de Sá 299A, Icarai, Niterói, RJ.

** Estudante de graduação/UFF.Rua Cândido Gaffré 205/31, Urca, Rio de Janeiro, RJ.

*** Departamento de Patologia e Clínica Veterinária.Faculdade de Veterinária, UFF. Rua Vital Brazil Filho, 64, Santa Rosa, Niterói, RJ, CEP 24230-340.
} 
sutis ou inexistentes (Tonelli, 1989; Atkins et al., 1997) e os sinais clínicos e achados físicos muitas vezes são inconstantes ou inespecíficos (Atkins et al., 1995). Com o desenvolvimento de testes mais sensíveis, o diagnóstico passou a ser mais facilmente estabelecido (Knight et al., 1999). Ainda não é possível saber se gatos portadores de infecções ectópicas com migração pelo sistema nervoso, por exemplo, apresentam ou não antígenos de parasitas adultos ou anticorpos na circulação. No entanto, como os parasitas podem não ser detectados à necrópsia, o resultado pode sugerir que o resultado do exame imunológico tenha sido falso positivo (McCall et al., 1995; Brown et al., 1999).

A maioria dos testes que visa a identificação de antígenos de parasitas adultos só é capaz de detectar infecções quando o hospedeiro alberga mais de uma fêmea adulta, com mais de oito meses de idade (Dillon, 1986; Tonelli, 1989; McCall et al., 1992; McCall et al., 1995; Atkins et al., 1997; Knight et al., 1999). Essa limitação dos testes torna o diagnóstico da infecção felina difícil, uma vez que gatos não costumam albergar mais de dois vermes e por pouco tempo (Tonelli, 1989; McCall et al., 1994; Atkins et al., 1995; Atkins et al., 1997; Knight et al., 1999). Como nas infecções felinas a carga parasitária é baixa e geralmente com menos de duas fêmeas adultas (McCall et al., 1992; Holmes, 1993; Mansour et al., 1995; McCall et al., 1995 Selcer et al., 1996; Atkins et al., 1997; Knight et al., 1999), há grandes chances de se obter resultados falso negativos (Tonelli, 1989; Larsson et al., 1992; McCall et al., 1992; Selcer et al., 1996; Venco et al., 1998; Knight et al., 1999). No entanto, existem relatos de que o período de detecção do antígeno de $D$. immitis pode variar de acordo com o teste utilizado (McCall, 1999). Quando o hospedeiro elimina fêmeas adultas, o resultado do teste pode permanecer positivo durante algumas semanas, podendo chegar a quatro meses (Knight et al., 1999).

Testes sorológicos para detecção de anticorpos contra $D$. immitis são mais sensiveis do que os testes para a detecção de antígenos (Wong et al., 1983; McCall et al., 1994; Atkins et al., 1995; Atkins et al., 1997; Dillon et al., 1997b), apesar de menos específicos, o que levoú ao isolamento de um gen comum a parasitas machos e fêmeas que codifica um antígeno recombinante (rHWAg1) exclusivo de D. immitis. É contra esse antígeno que os anticorpos detectados pelos testes atuais são dirigidos (Dillon et al., 1997b). Anticorpos podem ser desenvolvidos em resposta a larvas infectantes que podem não chegar à fase adulta. Logo, um resultado que demonstre a presença de anticorpos representa forte evidência de que este animal tenha entrado em contato com o agente etiológico mas não representa, necessariamente, que ele é um portador de vermes adultos. Um resultado negativo demostra que o animal provavelmente não foi exposto ao nematóide ou eliminou-o naturalmente há mais de oito meses, tempo necessário para que o animal soro-converta para a categoria de anticorpo negativo. Pode ser ainda que a infecção tenha ocorrido há pouco tempo, o que dificulta sua detecção (Atkins et al., 1995; McCall et al., 1995; Dillon et al., 1997b; Atkins et al., 1997; Knight et al., 1999; McCall, 1999; Piché, 1999). Nos casos em que as larvas infectantes são eliminadas antes de dois meses após a infecção são necessárias inúmeras infecções para que anticorpos sejam produzidos em niveis detectáveis pelo teste do laboratório Heska (Donoughue et al., 1999).
O diagnóstico de infecções em gatos deve ser realizado em duas etapas, primeiro, a detecção de anticorpos e, posteriormente, a deteç̧ão de antígenos de parasitas adultos. A detecção de anticorpos contra $D$. immitis pode ser realizada três meses após a infecção em gatos, e ao se utilizar o teste Heska, dois meses após a infecção aproximadamente 15\% dos animais positivos podem ser detectados, no entanto já foram observados diagnósticos a partir de um mês após infecção ao utilizar os testes dos laboratórios Antech (24\%) (imunofluorescência indireta) e Animal Diagnostics' (19\%) (ELISA) (McCall, 1999). A diferença de sensibilidade existente entre os testes comerciais geralmente só afeta detecçōes de infecções em animais com baixo nível de antigenos. A baixa antigenemia está usualmente associada a infecções leves que provavelmente se tornarão detectáveis durante o curso da patologia (Knight et al., 1999).

\section{Material e métodos}

Foram obtidas amostras de sangue de 77 cães acima de um ano de idade, independentemente de suspeita clínica, residentes no Engenho no Mato, Niterói, RJ, por punção da veia cefálica, durante a campanha municipal de vacinação contra raiva, em setembro de 1998. No laboratório as amostras foram submetidas à técnica de Knott (1934) modificada por Newton e Wright (1956) para pesquisa de microfilárias de $D$. immitis.

Foi realizada coleta de sangue de 55 gatos (26 fêmeas, 29 machos) com mais de um ano de idade, independentemente de suspeita clínica, residentes em um gatil no Engenho do Mato, Niterói, RJ, entre julho e novembro de 1998. Os animais foram sedados (22mg/kg ketamina e $0,044 \mathrm{mg} / \mathrm{kg}$ atropina) e o sangue obtido por punção jugular. As amostras foram levadas para o laboratório e com o plasma, foi realizado o teste de ELISA indireto do laboratório Heska' para a identificação de anticorpos contra o antígeno (rHWAg1) do parasita $D$. immitis.

\section{Resultados e discussão}

Dos 77 cães examinados quanto à presença de microfilárias no sangue, 20 apresentavam larvas ha circulação (26\%) e nenhum dos 55 gatos apresentou anticorpos contra $D$. immitis na circulação.

A identificação de cães portadores de microfilaremia (26\%) no Engenho do Mato, Niterói, RJ, demonstrou que havia fonte de infecção para mosquitos vetores na regiäo onde se localiza o gatil estudado e, conseqüentemente, demonstra a possibilidade de haver transmissão de $D$. immitis no bairro.

Nenhum dos gatos examinados apresentou anticorpos contra $D$. immitis na circulação, embora todos os animais já habitassem o local há mais de um ano. É provável que eles não apresentassem anticorpos porque são necessárias várias infecções malsucedidas para que o nivel de anticorpos seja detectável no teste usado e o período durante o qual esses anticorpos são detectáveis é curto, aproximadamente 100 dias (Donoghue et al., 1999). Mesmo quando larvas infectantes transmitidas por mosquitos desenvolvem-se em felinos, só há detecção de anticorpos a partir de dois meses pós-infec-

\footnotetext{
${ }^{1}$ Heska - Partida V0066k32101.
} 
ção (15\% dos casos). Na maioria dos casos, somente a partir de três meses pós-infecção (McCall, 1999), dificultando ainda mais o diagnóstico. Caso um animal tenha sido infectado e albergado parasitas adultos, e esses vermes tenham morrido, os anticorpos desaparecem no período de oito a nove meses (Dillon et al., 1997a; Dillon et al., 1997b). $\mathrm{Na}$ realidade, o fato de anficorpos não terem sido encontra-

\section{Agradecimentos}

Secretaria Municipal de Saúde de Niterói.

\section{Referências}

ATKINS, C. E., ATWELL, R. B., DILLON, R., GENCHI, C., HAYASAKI, M., HOLMES, R. A., KNIGHT, D. H., LUKOF, D. K., MCCALL, J. W., SLOCOMBE, J. O. D. Guidelines for the diagnosis, treatment, and prevention of heartworm (Dirofilaria immitis) infection in cats. Proceedings of the Heartworm Symposium'95, Auburn, Alabama, 1995, p. 309-312.

ATKINS, C. E., ATWELL, R. B., DILLON, R., GENCHI, C., HAYASAKI, M., HOLMES, R. A., KNIGHT, D. H., LUKOF, D. K., MCCALL, J. W., SLOCOMBE, J. O. D. Guidelines for the diagnosis, treatment and prevention of heartworm (Dirofilaria immitis) infection in cats. Compendium of Continuing Education - Small Animal Parasitology, 1997, p. 422-429.

BROWN, W., PAUL, A, VENCO, L., MCCALL, J., BRUNT, J. Feline Heartworm Disease. Feline Practice, v. 27, n. 1, 1999.

DILLON, A. R. Feline heartworm disease. Proceedings of the Heartworm Symposium'86, New Orleans, LA., 1986, p. 149-154.

DILLON, A. R., ATKINS, C., CLEKIS, T., GENCHI, C., KNIGHT, D. H., MCCALL, J., MILLER, M. Feline Heartworm Disease, Part 1. Feline Practice, v. 24, n. 6, p. 12-16, 1996.

DILLON, A. R., ATKINS, C., CLEKIS, T., GENCHI, C., KNIGHT, D. H., MCCALL, J., MILLER, M. W. Feline Heartworm Disease, Part 2. Feline Practice, v. 25, n. 1, p.26-30, 1997a.

DILLON, A. R., ATKINS, C., CLEKIS, T., GENCHI, C., KNIGHT, D. H., MCCALL, J., MILLER, M. Feline Heartworm Disease, Part 3. Feline Practice, v. 25, n. 2, p. 12-21, 1997b

DONOGHUE, AR.; PICHÉ, C. A; RADECKI, STEVEN, V., SCHAAD, L. E.; FRANK, G. R. Effect of Prophylaxis on Antiheartworm Antibody Levels in Cats Receiving Trickle Experimental Infeccions of Dirofilaria immitis. Proceedings of the Heartworm Symposium'99, Tampa Florida, 1999, p. 135-138.

GUERRERO, J., MCCALL, J. W., DZIMIANSKI, M. T., MCTIER, T. L., HOLMES, R. A. NEWCOMB K. M. Prevalence of Dirofilaria immitis infection in cats from the Southeastern United States. In Proceedings of the Heartworm Symposium' 92, Austin, TX, 1992, p. 91-95.

HOLMES, R. A. Feline Heartworm Disease. Small Animal Parasitology, v. 15, n. 5, p. $687-695,1993$

KNIGHT, D. T, ATKINS, C. E., ATWEL, R. B., COURTNEY, C. H., DILLON. R., GENCHI, C., HAGIO, M., HOLMES, R. A, LUKOF, D. K., MCCALL, J. W., VENCO, L. Guidelines for the diagnosis, treatment, and prevention of heartworm. American Heartworm Society Bulletin, v. 26, n. 6, 1999. KNOTT, J. A method for making microfilarial survey on dog blood. Trans. R. Soc. Trop. Med. Hyg, v. 33, p.186-191, 1939. dos sugere que nenhum dos gatos, apesar de expostos, albergasse vermes com mais de três meses pós-infecção. Entretanto, não significa que esses gatos não tivessem sido infectados esporadicamente e eliminado as larvas naturalmente, o que demonstra que mais estudos são necessários para esclarecimento do risco de infecção por $D$. immitis para felinos na região de Engenho do Mato, Niterói, RJ.
MANSOUR, A E., MCCALL, J. W., MCTIER, T. L., SUPAKORNDEJ, N., RICKETTS, R. Epidemiology of feline heartworm infection: laboratory studies on transmission and on host preference of mosquito vectors. Proceedings of Heartworm Symposiun'95, Auburn Alabama, 1995, p. 87-95.

MCCALL, J. W., DZIMIANSKI, M. T., MCTIER, T.L., JERNIGAN, A. D., JUN, J. J., MANSOUR, A. E., SUPAKORNDEJ, N., PLUE, R. E., CLARK, J. N., WALLACE, D. H., LEWIS, R. E. Biology of Experimental Heartworm Infections in Cats. Proceedings of Heartworm Symposiun'92, Austin Texas.1992, p. 27-29/71-79.

MCCALL, J. W, CALVERT, C. A. , RAWLINGS, C. A. Heartworm infection in cats: A life-threatening disease. Symposium on Treating Heartworm Infection, 1994, p. 639-647.

MCCALL, J. W., SUPAKORNDEJ, N., RYAN, W., SOLL, M. D. Utility of an ELISA-based Antibody Test for Detection of Heartworm Infection in Cats. Proceedings of Heartworm Symposiun'95, Auburn Alabama, 1995, p. 127-133.

McCALL, J. W. Evaluation of the Accuracy of Heartworm Antigen and Antibody Tests for Cats. Recent Advances in Heartworm Disease: Symposium'98, Tampa Florida, 1999, p. 127-134.

NEWTON, W. L., WRIGHT, W. H. The occurrence of a dog filariid other than Dirofilaria immitis in the United States. J. Parasitol., v. 42, p. 246 256, 1956

PICHÉ, C. A. Results of antibody and antigen testing for feline heartworm infection at HeskaÒ veterinary diagnostic laboratories. Recent Advances in Heartworm Disease: Symposium'98, p. 139-143, 1999.

RYAN, W. G., GROSS, S. J., SOLL, M. D. Diagnosis of Feline Heartworm Infection. Proceedings of Heartworm Symposiun'95, Auburn, Alabama, 1995, p. 121-126.

SCHREY, C. F., TRAUTVETTER, E. Dirofilariosis canina y felina-diagnóstico y tratamiento. Waltham Focus, v.8, n. 2, p. 24-30, 1998.

SELCER, B. A., NEWELL, S. M., MANSOUR, A. E., MCCALL, J. W. Radiographic and 2-D Echocardiographic findings in eighteen cats experimentally exposed to $D$. immitis via mosquito bites. Feline Heartworm Disease, v. 37, n. 1, p. 37-44, 1996.

TONELLI, Q. J. Factors Affecting the Accuracy of Enzyme Immunoassays for Dirofilaria immitis Adult Antigen. Proceedings of Heartworm Symposium'89, Charleston South Carolina, 1989, p. 161-165

VENCO, L., CALZOLARI, D., MAZZOCCHI, D., MORINI, S., GENCHI, C. The Use of Echocardiography as a Diagnostic Tool for Detection of Feline Heartworm (Dirofilaria immitis) Infections. Feline Practice, v. 26. n. 3, p. 6-9, 1998.

WONG, M. M., PEDERSEN, N. C., CULLEN, J. Dirofilariasis in cats. J.A.V.M.A., v. 19, n. 6, p. 855-864, 1983 